Mathematical Sciences and Applications

E-NOTES

MATHEMATICAL

SCIENCES

AND APPLICATIONS

E-NOTES

https://doi.org/10.36753/mathenot.641660

9 (1) 36-41 (2021) - Research Article

ISSN: 2147-6268

(C)MSAEN

\title{
Reciprocal Complementary Distance Energy of Complement of Line Graphs of Regular Graphs
}

\author{
Harishchandra S. Ramane* and B. Parvathalu
}

\begin{abstract}
The reciprocal complementary distance $(R C D)$ matrix of a graph $G$ is defined as $R C D(G)=\left[r_{i j}\right]$, where $r_{i j}=\frac{1}{1+D-d_{i j}}$ if $i \neq j$ and $r_{i j}=0$, otherwise, where $D$ is the diameter of $G$ and $d_{i j}$ is the distance between the vertices $v_{i}$ and $v_{j}$ in $G$. The $R C D$-energy of $G$ is defined as the sum of the absolute values of the eigenvalues of $R C D$-matrix. Two graphs are said to be $R C D$-equienergetic if they have same $R C D$-energy. In this paper, the $R C D$-energy of the complement of line graphs of certain regular graphs in terms of the order and degree is obtained and as a consequence, pairs of $R C D$-equienergetic graphs of same order and having different $R C D$-eigenvalues are constructed.
\end{abstract}

Keywords: Reciprocal complementary distance ( $R C D)$ eigenvalues; $R C D$-energy of a graph; $R C D$-equienergetic graphs. AMS Subject Classification (2020): Primary: 05C50

*Corresponding author

\section{Introduction}

Let $G$ be a simple, undirected, connected graph with $n$ vertices and $m$ edges. Let the vertex set of $G$ be $V(G)=\left\{v_{1}, v_{2}, \ldots, v_{n}\right\}$. The adjacency matrix of a graph $G$ is the square matrix $A(G)=\left[a_{i j}\right]$ of order $n$, in which $a_{i j}=1$ if $v_{i}$ is adjacent to $v_{j}$ and $a_{i j}=0$, otherwise. The eigenvalues of $A(G)$ are the adjacency eigenvalues of $G$, and they are labeled as $\lambda_{1} \geq \lambda_{2} \geq \cdots \geq \lambda_{n}$. Two non-isomorphic graphs are said to be adjacency cospectral or simply cospectral if they have same adjacency eigenvalues [3].

The distance between the vertices $v_{i}$ and $v_{j}$, denoted by $d_{i j}$, is the length of the shortest path joining $v_{i}$ and $v_{j}$. The diameter of a graph $G$, denoted by $\operatorname{diam}(G)$, is the maximum distance between any pair of vertices of $G$. A graph $G$ is said to be $r$-regular graph if all of its vertices have same degree equal to $r$. The complement of a graph $G$, denoted by $\bar{G}$, is a graph with vertex set $V(G)$ and two vertices in $\bar{G}$ are adjacent if and only if they are not adjacent in $G$. The line graph of $G$, denoted by $L(G)$ is the graph whose vertices corresponds to the edges of $G$ and two vertices of $L(G)$ are adjacent if and only if the corresponding edges are adjacent in $G$. For $k=1,2, \ldots$, the $k$-th iterated line graph of $G$ is defined as $L^{k}(G)=L\left(L^{k-1}(G)\right)$, where $L^{0}(G)=G$ and $L^{1}(G)=L(G)$ [5].

The line graph of a regular graph $G$ of order $n_{0}$ and of degree $r_{0}$ is a regular graph of order $n_{1}=\left(n_{0} r_{0}\right) / 2$ and 
of degree $r_{1}=2 r_{0}-2$. Consequently the order and degree of $L^{k}(G)$ are $[1,2]$

$$
n_{k}=\frac{r_{k-1} n_{k-1}}{2}
$$

and

$$
r_{k}=2 r_{k-1}-2
$$

where $n_{i}$ and $r_{i}$ stands for order and degree of $L^{i}(G), i=0,1, \ldots$

Therefore

$$
r_{k}=2^{k} r_{0}-2^{k+1}+2
$$

and

$$
n_{k}=\frac{n_{0}}{2^{k}} \prod_{i=0}^{k-1} r_{i}=\frac{n_{0}}{2^{k}} \prod_{i=0}^{k-1}\left(2^{i} r_{0}-2^{i+1}+2\right) .
$$

The reciprocal complementary distance matrix or $R C D$-matrix $[6,8]$ of a graph $G$ is an $n \times n$ matrix $R C D(G)=\left[r_{i j}\right]$, where

$$
r_{i j}=\left\{\begin{array}{ccc}
\frac{1}{1+D-d_{i j}} & \text { if } & i \neq j \\
0 & \text { if } & i=j,
\end{array}\right.
$$

where $D$ is the diameter of $G$ and $d_{i j}$ is the distance between the vertices $v_{i}$ and $v_{j}$ in $G$.

The reciprocal complementary distance matrix is an important source of structural descriptors in the quantitative structure property relationship (QSPR) model in chemistry [6, 8].

The eigenvalues of $R C D(G)$, labeled as $\mu_{1} \geq \mu_{2} \geq \cdots \geq \mu_{n}$ are said to be the reciprocal complementary distance eigenvalues or $R C D$-eigenvalues of $G$ and their collection is called $R C D$-spectra of $G$. Two non-isomorphic graphs are said to be $R C D$-cospectral if they have same $R C D$-spectra.

The reciprocal complementary distance energy or $R C D$-energy of a graph $G$, denoted by $R C D E(G)$, is defined as [11]

$$
\operatorname{RCDE}(G)=\sum_{i=1}^{n}\left|\mu_{i}\right|
$$

The Eq. (1.5) is defined in full analogy with the ordinary graph energy $E(G)$, defined as [4]

$$
E(G)=\sum_{i=1}^{n}\left|\lambda_{i}\right|
$$

where $\lambda_{1}, \lambda_{2}, \ldots, \lambda_{n}$ are the adjacency eigenvalues of $G$. The ordinary graph energy has a relation with the total $\pi$-electron energy of a molecule in quantum chemistry [9].

Two connected graphs $G_{1}$ and $G_{2}$ are said to be reciprocal complementary distance equienergetic or $R C D$-equienergetic if $R C D E\left(G_{1}\right)=R C D E\left(G_{2}\right)$. In [10,11] $R C D$-equienergetic graphs are obtained. In this paper we obtain the $R C D$-energy of the complement of iterated line graphs of certain regular graphs and thus give another construction of $R C D$-equienergetic graphs having different $R C D$-spectra.

We need following results.

Theorem 1.1. [3] If $G$ is an $r$-regular graph, then its maximum adjacency eigenvalue is equal to $r$.

Theorem 1.2. [13] If $\lambda_{1}, \lambda_{2}, \ldots, \lambda_{n}$ are the adjacency eigenvalues of a regular graph $G$ of order $n$ and of degree $r$, then the adjacency eigenvalues of $L(G)$ are

$$
\begin{aligned}
\lambda_{i}+r-2, & i=1,2, \ldots, n, \quad \text { and } \\
-2, & n(r-2) / 2 \text { times. }
\end{aligned}
$$

Theorem 1.3. [12] Let $G$ be an r-regular graph of order $n$. If $r, \lambda_{2}, \ldots, \lambda_{n}$ are the adjacency eigenvalues of $G$, then the adjacency eigenvalues of $\bar{G}$ are $n-r-1$ and $-\lambda_{i}-1, i=2,3, \ldots, n$.

Theorem 1.4. [11] Let $G$ be an $r$-regular graph on $n$ vertices and $\operatorname{diam}(G)=2$. If $r, \lambda_{2}, \ldots, \lambda_{n}$ are the adjacency eigenvalues of $G$, then its $R C D$-eigenvalues are $n-1-\frac{r}{2}$ and $-1-\frac{\lambda_{i}}{2}, i=2,3, \ldots, n$.

Lemma 1.1. [7] Let $G$ be an $r$-regular graph on $n$ vertices. If $r \leq \frac{n-1}{2}$ then $\operatorname{diam}\left(\overline{L^{k}(G)}\right)=2, k \geq 1$. 


\section{2. $R C D$-Energy}

Theorem 2.1. Let $G$ be a regular graph of order $n$ and degree $r \geq 4$. If $r \leq \frac{n-1}{2}$, then

$$
R C D E\left(\overline{L^{2}(G)}\right)=\frac{3 n r}{2}(r-2) .
$$

Proof. Let the adjacency eigenvalues of $G$ be $r, \lambda_{2}, \ldots, \lambda_{n}$. By Theorem 1.2, the adjacency eigenvalues of $L(G)$ are

$$
\left.\begin{array}{rl}
2 r-2, & \text { and } \\
\lambda_{i}+r-2, & i=2,3, \ldots, n, \quad \text { and } \\
-2, & n(r-2) / 2 \text { times. }
\end{array}\right\}
$$

Since $L(G)$ is a regular graph of order $n r / 2$ and of degree $2 r-2$, by Theorem 1.2 and Eq. (2.1), the adjacency eigenvalues of $L^{2}(G)$ are

$$
\begin{aligned}
4 r-6, & \text { and } \\
\lambda_{i}+3 r-6, & i=2,3, \ldots, n, \\
2 r-6, & n(r-2) / 2, \\
-2, & n r(r-2) / 2 \text { times. }
\end{aligned}
$$

From Theorem 1.3 and Eq. (2.2), the adjacency eigenvalues of $\overline{L^{2}(G)}$ are

$$
\left.\begin{array}{rll}
(n r(r-1) / 2)-4 r+5, & \text { and } & \\
-\lambda_{i}-3 r+5, & i=2,3, \ldots, n, & \text { and } \\
-2 r+5, & n(r-2) / 2, & \text { and } \\
1, & n r(r-2) / 2 \text { times. } &
\end{array}\right\}
$$

The graph $\overline{L^{2}(G)}$ is a regular graph of order $n r(r-1) / 2$ and of degree $(n r(r-1) / 2)-4 r+5$. Since $r \leq \frac{n-1}{2}$, by Lemma 1.1, $\operatorname{diam}\left(\overline{L^{2}(G)}\right)=2$. Therefore by Theorem 1.4 and Eq. (2.3), the $R C D$-eigenvalues of $\overline{L^{2}(G)}$ are

$$
\left.\begin{array}{rlr}
\left(n r^{2}-n r+8 r-14\right) / 4, & \text { and } & \\
\left(\lambda_{i}+3 r-7\right) / 2, & i=2,3, \ldots, n, & \text { and } \\
(2 r-7) / 2, & n(r-2) / 2, & \text { and } \\
-(3 / 2), & n r(r-2) / 2 \text { times. } &
\end{array}\right\}
$$

All adjacency eigenvalues of a regular graph of degree $r$ satisfy the condition $-r \leq \lambda_{i} \leq r$ [3].

If $r \geq 4$, then $\left(n r^{2}-n r+8 r-14\right) \geq 0, \lambda_{i}+3 r-7 \geq 0$ and $2 r-7 \geq 0$.

Therefore by Eq. (2.4),

$$
\begin{aligned}
\operatorname{RCDE}\left(\overline{L^{2}(G)}\right)= & \frac{n r^{2}-n r+8 r-14}{4}+\sum_{i=2}^{n} \frac{\left(\lambda_{i}+3 r-7\right)}{2} \\
& +\left(\frac{2 r-7}{2}\right) \frac{n(r-2)}{2}+\left|-\frac{3}{2}\right| \frac{n r(r-2)}{2} \\
= & \frac{3 n r}{2}(r-2) \quad \text { since } \quad \sum_{i=2}^{n} \lambda_{i}=-r .
\end{aligned}
$$


Corollary 2.1. Let $G$ be a regular graph of order $n_{0}$ and of degree $r_{0} \geq 4$. Let $n_{k}$ and $r_{k}$ be the order and degree respectively of the $k$-th iterated line graph $L^{k}(G), k \geq 2$. If $r_{0} \leq \frac{n_{0}-1}{2}$, then

$$
\operatorname{RCDE}\left(\overline{L^{k}(G)}\right)=\frac{3 n_{k-2} r_{k-2}}{2}\left(r_{k-2}-2\right) .
$$

Proof. If $r_{0} \leq \frac{n_{0}-1}{2}$, then by Eqs. (1.1) and (1.2), we have

$$
r_{1}=2 r_{0}-2 \leq n_{0}-3 \leq \frac{1}{2}\left(\frac{n_{0} r_{0}}{2}-1\right)=\frac{n_{1}-1}{2} .
$$

Hence

$$
r_{k-2} \leq \frac{n_{k-2}-1}{2}
$$

Therefore by Theorem 2.1,

$$
\operatorname{RCDE}\left(\overline{L^{k}(G)}\right)=\operatorname{RCDE}\left(\overline{L^{2}\left(L^{k-2}(G)\right)}\right)=\frac{3 n_{k-2} r_{k-2}}{2}\left(r_{k-2}-2\right) .
$$

Corollary 2.2. Let $G$ be a regular graph of order $n_{0}$ and of degree $r_{0} \geq 4$. Let $n_{k}$ and $r_{k}$ be the order and degree respectively of the $k$-th iterated line graph $L^{k}(G), k \geq 2$. If $r_{0} \leq \frac{n_{0}-1}{2}$, then

$$
\operatorname{RCDE}\left(\overline{L^{k}(G)}\right)=\frac{3}{2} n_{0}\left(r_{0}-2\right) \prod_{i=0}^{k-2}\left(2^{i} r_{0}-2^{i+1}+2\right)
$$

Theorem 2.2. Let $G$ be a cubic graph of order $n \geq 7$. Then

$$
\operatorname{RCDE}(\overline{L(G)})=\frac{3 n+E(G)}{2} .
$$

Proof. Let the adjacency eigenvalues of $G$ be $3, \lambda_{2}, \ldots, \lambda_{n}$. From Theorem 1.2, the adjacency eigenvalues of $L(G)$ are

$$
\left.\begin{array}{rl}
4, & \text { and } \\
\lambda_{i}+1, & i=2,3, \ldots, n, \quad \text { and } \\
-2, & n / 2 \text { times. }
\end{array}\right\}
$$

From Theorem 1.3 and the Eq. (2.5), the adjacency eigenvalues of $\overline{L(G)}$ are

$$
\left.\begin{array}{rl}
(3 n / 2)-5, & \text { and } \\
-\lambda_{i}-2, & i=2,3, \ldots, n, \quad \text { and } \\
1, & n / 2 \text { times. }
\end{array}\right\}
$$

Since $G$ is a cubic graph on $n \geq 7$ vertices, $3 \leq \frac{n-1}{2}$. Therefore by Lemma 1.1, $\operatorname{diam}(\overline{L(G)})=2$.

Therefore by Theorem 1.4 and Eq. (2.6), the $R C D$-eigenvalues of $\overline{L(G)}$ are

Therefore

$$
\left.\begin{array}{rl}
(3 n+6) / 4, & \text { and } \\
\frac{\lambda_{i}}{2}, & i=2,3, \ldots, n, \quad \text { and } \\
(-3 / 2), & n / 2 \text { times. }
\end{array}\right\}
$$

$$
\begin{aligned}
\operatorname{RCDE}(\overline{L(G)}) & =\left|\frac{3 n+6}{4}\right|+\sum_{i=2}^{n}\left|\frac{\lambda_{i}}{2}\right|+\left|-\frac{3}{2}\right| \frac{n}{2} \\
& =\frac{3 n}{4}+\frac{3}{2}+\frac{1}{2}(E(G)-3)+\frac{3 n}{4} \\
& =\frac{3 n+E(G)}{2} .
\end{aligned}
$$




\section{3. $R C D$-Equienergetic graphs}

If $G_{1}$ and $G_{2}$ are two regular graphs of same order and of same degree, then by Eq. (1.3) and (1.4) for any $k \geq 1$, $L^{k}\left(G_{1}\right)$ and $L^{k}\left(G_{2}\right)$ are also regular graphs of the same order and have the same number of edges. Hence $\overline{L^{k}\left(G_{1}\right)}$ and $\overline{L^{k}\left(G_{2}\right)}$ are regular graphs of the same order and have the same number of edges.

Proposition 3.1. Let $G_{1}$ and $G_{2}$ be regular graphs of the same order $n$ and of the same degree $r$. If $r \leq \frac{n-1}{2}$, then for $k \geq 1$, $\overline{L^{k}\left(G_{1}\right)}$ and $\overline{L^{k}\left(G_{2}\right)}$ are $R C D$-cospectral if and only if $G_{1}$ and $G_{2}$ are cospectral.

Proof. If $G_{1}$ and $G_{2}$ are regular cospectral graphs then applying Theorem 1.2 repeatedly we get that $L^{k}\left(G_{1}\right)$ and $L^{k}\left(G_{2}\right)$ are cospectral for $k \geq 1$. Therefore by Theorem 1.3, $\overline{L^{k}\left(G_{1}\right)}$ and $\overline{L^{k}\left(G_{2}\right)}$ are cospectral. Since $r \leq \frac{n-1}{2}$, by Lemma 1.1, $\operatorname{diam}\left(\overline{L^{k}\left(G_{1}\right)}\right)=2$ and $\operatorname{diam}\left(\overline{L^{k}\left(G_{2}\right)}\right)=2$. Therefore by Theorem $1.4, \overline{L^{k}\left(G_{1}\right)}$ and $\overline{L^{k}\left(G_{2}\right)}$ are $R C D$-cospectral.

Conversely, let $\overline{L^{k}\left(G_{1}\right)}$ and $\overline{L^{k}\left(G_{2}\right)}$ are $R C D$-cospectral. Suppose $G_{1}$ and $G_{2}$ are not cospectral. Then by Theorem 1.2, $L^{k}\left(G_{1}\right)$ and $L^{k}\left(G_{2}\right)$ are not cospectral for $k \geq 1$. Hence by Theorem $1.3, \overline{L^{k}\left(G_{1}\right)}$ and $\overline{L^{k}\left(G_{2}\right)}$ are not cospectral. Now, by using Theorem $1.4, \overline{L^{k}\left(G_{1}\right)}$ and $\overline{L^{k}\left(G_{2}\right)}$ are not $R C D$-cospectral, which is a contradiction. Hence $G_{1}$ and $G_{2}$ are cospectral.

Theorem 3.1. Let $G_{1}$ and $G_{2}$ be regular, not cospectral graphs of the same order $n$ and of the same degree $r \geq 4$. If $r \leq \frac{n-1}{2}$, then $\overline{L^{2}\left(G_{1}\right)}$ and $\overline{L^{2}\left(G_{2}\right)}$ form a pair of not $R C D$-cospectral, $R C D$-equienergetic graphs of equal order and of equal number of edges.

Proof. If $G_{1}$ and $G_{2}$ are regular, not cospectral graphs of the same order $n$, same degree $r \geq 4$ and $r \leq \frac{n-1}{2}$, then by Proposition 3.1, $\overline{L^{2}\left(G_{1}\right)}$ and $\overline{L^{2}\left(G_{2}\right)}$ form a pair of not $R C D$-cospectral graphs of same order and same size. And by Theorem 2.1, $R C D E\left(\overline{L^{2}\left(G_{1}\right)}\right)=\frac{3 n r}{2}(r-2)=R C D E\left(\overline{L^{2}\left(G_{2}\right)}\right)$, which implies that $\overline{L^{2}\left(G_{1}\right)}$ and $\overline{L^{2}\left(G_{2}\right)}$ form a pair $R C D$-equienergetic graphs.

Theorem 3.2. Let $G_{1}$ and $G_{2}$ be regular, not cospectral graphs of the same order $n$ and of the same degree $r \geq 4$. If $r \leq \frac{n-1}{2}$, then for $k \geq 2, \overline{L^{k}\left(G_{1}\right)}$ and $\overline{L^{k}\left(G_{2}\right)}$ form a pair of not $R C D$-cospectral, $R C D$-equienergetic graphs of equal order and of equal number of edges.

Proof. Since $\overline{L^{k}\left(G_{1}\right)}=\overline{L^{2}\left(L^{k-2}\left(G_{1}\right)\right)}$ and $\overline{L^{k}\left(G_{2}\right)}=\overline{L^{2}\left(L^{k-2}\left(G_{2}\right)\right)}$, the result follows from Theorem 3.1.

Proposition 3.2. Let $G_{1}$ and $G_{2}$ be cubic graphs of order $n \geq 7$, such that $E\left(G_{1}\right)=E\left(G_{2}\right)$. Then

$$
\operatorname{RCDE}\left(\overline{L\left(G_{1}\right)}\right)=\operatorname{RCDE}\left(\overline{L\left(G_{2}\right)}\right) .
$$

Proof. The result follows from Theorem 2.2 as $E\left(G_{1}\right)=E\left(G_{2}\right)$.

\section{Acknowledgment:}

The authors would like to thank the anonymous referee(s) for valuable comments which helped to improve the manuscript. The author HSR is thankful to the University Grants Commission (UGC), New Delhi, for support through UGC-SAP DRS-III, 2016-2021: F.510/3/ DRS-III / 2016 (SAP-I).

\section{References}

[1] Buckley, F.: Iterated line graphs. Congr. Numer. 33, 390-394 (1981).

[2] Buckley, F.: The size of iterated line graphs. Graph Theory Notes of New York. 25, 33-36 (1993).

[3] Cvetković, D., Rowlinson, P., Simić, S.: Introduction to the Theory of Graph Spectra. Cambridge University Press. Cambridge (2010).

[4] Gutman, I.: The energy of a graph. Ber. Math. Stat. Sekt. Forschungsz. Graz. 103, 1-22 (1978).

[5] Harary, F.: Graph Theory. Addison-Wesley Publishing Co., Reading (1969). 
[6] Ivanciuc, O., Ivanciuc, T., Balaban, A. T.: The complementary distance matrix, a new moleculargraph metric. ACHModels Chem. 137, 57-82 (2000).

[7] Indulal, G.: D-spectrum and D-energy of complements of iterated line graphs of regular graphs. J. Alg. Stru. Appl. 4, 51-56 (2017). https:/ / doi.org/10.29252/asta.4.1.51

[8] Jenežić, D., Miličević, A., Nikolić, S., Trinajstić, N.: Graph Theoretical Matrices in Chemistry. University of Kragujevac. Kragujevac (2007). https:/ / doi.org/10.1021/ci700278s

[9] Li, X., Shi, Y., Gutman, I.: Graph Energy. Springer. New York (2012). https://doi.org/10.1007/978-1-4614-4220-2

[10] Ramane, H. S., Gudodagi, G. A.: Reciprocal complementary equienergetic graphs. Asian-European J. Math. 9, ID: 1650084, pages 15 (2016). https:/ / doi.org/10.1142/S1793557116500844

[11] Ramane, H. S., Yalnaik,A. S.: Reciprocal complementary distance spectra and reciprocal complementary distance energy of line graphs of regular graphs. El. J. Graph Theory Appl. 3, 228-236 (2015). http://dx.doi.org/10.5614/ejgta.2015.3.2.10

[12] Sachs, H.: Über selbstkomplementare Graphen. Publ. Math. Debrecen. 9, 270-288 (1962).

[13] Sachs, H.: Über Teiler, Faktoren und charakteristische Polynome von Graphen, Teil II. Wiss. Z. TH Ilmenau. 13, 405-412 (1967).

\section{Affiliations}

H. S. RAMANE

ADDRESS: Department of Mathematics, Karnatak University, Dharwad - 580003, India.

E-MAIL: hsramane@kud.ac.in

ORCID ID: 0000-0003-3122-1669

B. Parvathalu

AdDRESS: Department of Mathematics, Karnatak University's Karnatak Arts College, Dharwad - 580001, India. E-MAIL: bparvathalu@gmail.com

ORCID ID: 0000-0002-5151-8446 